\title{
VIDEO
}

\section{Salvage resection of recurrent polyp after polypectomy on the colorectal anastomosis using endoscopic submucosal dissection}

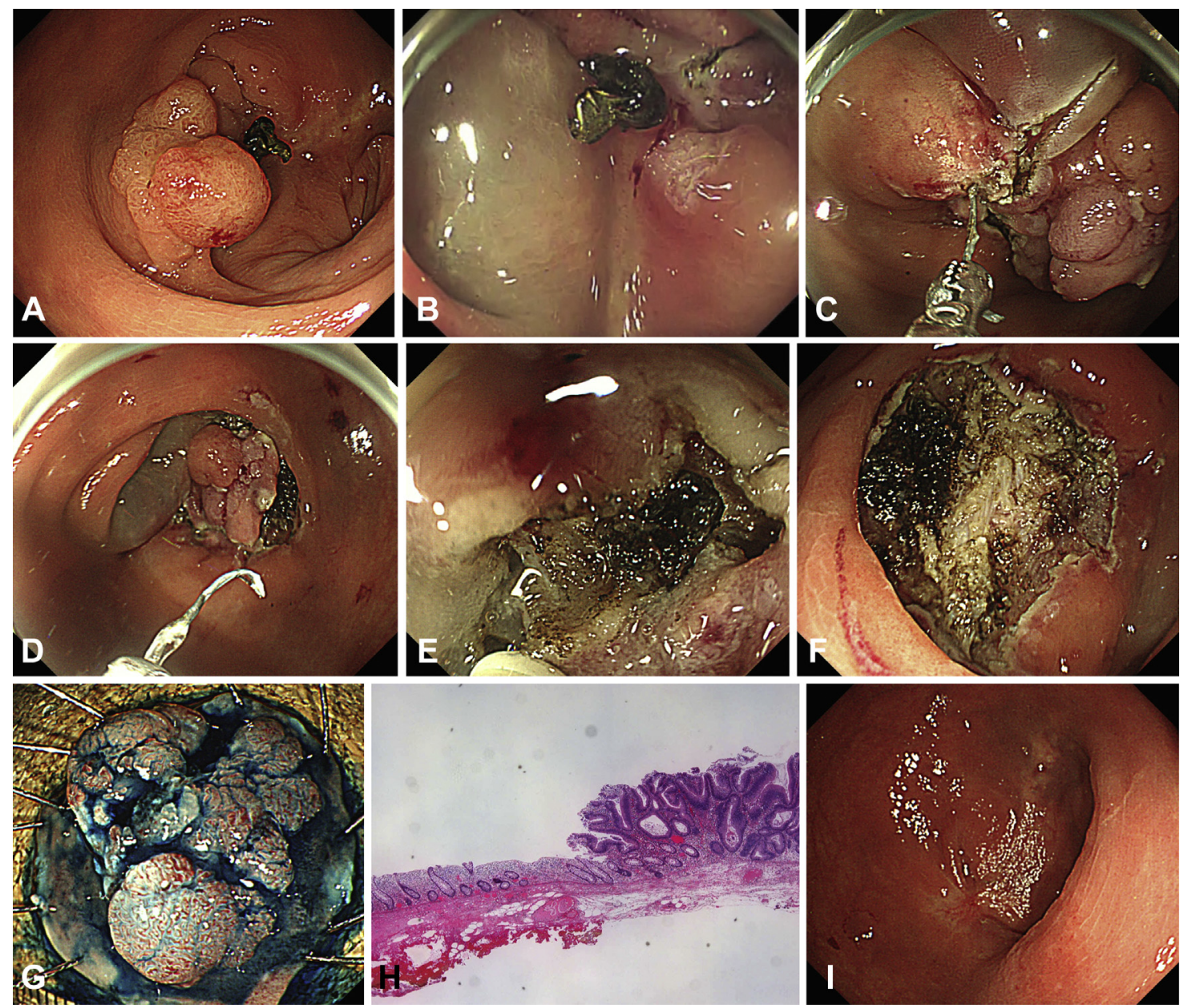

Figure 1. A, Recurrent polyp after polypectomy on the colorectal anastomosis. B, Surgical staple remaining in the incision line. C, D, Staple retrieved by biopsy forceps. E, Severe fibrosis in submucosal layer. F, Defect after resection. G, Resected lesion. H, Pathologic diagnosis was low-grade adenoma with fibrosis in the submucosal layer. I, ESD ulcer completely scarred 6 months later. ESD, endoscopic submucosal dissection.

Endoscopic submucosal dissection (ESD) enables secure R0 resection irrespective of size or location of the lesion. However, severe fibrosis is still a condition in which ESD is technically challenging. Here we report a case for which we performed salvage ESD for a recurrent polyp after polypectomy on the colorectal anastomosis.

A 77 -year-old woman who had undergone polypectomy for a polyp on the anastomosis of a previous laparoscopic sigmoidectomy was referred to our endoscopy unit for removal of its recurrence. Colonoscopy revealed a sessile polyp $15 \mathrm{~mm}$ in diameter on the anastomosis (Fig. 1A). We decided to perform ESD as a salvage treatment (Video 1, available online at www.VideoGIE.org). We started with a circumferential mucosal incision using dry cut mode effect 2, $30 \mathrm{~W}$, from the anal side, using a Dual Knife (KD-655Q; Olympus, Tokyo, Japan), followed by submucosal dissection using swift coagulation mode effect 3, $30 \mathrm{~W}$. A surgical staple remained on the incision line (Fig. 1B) and it was retrieved by biopsy forceps (Radial Jaw 4; Boston Scientific, Natick, Mass) (Figs. 1C 
and D). As expected, there was severe fibrosis in the submucosal layer (Fig. 1E), so we carefully dissected it using an ST hood (DH15-GR; Fujifilm, Tokyo, Japan) and Hook knife (KD-620QR; Olympus). During submucosal dissection, we encountered many staples, which we retrieved. Finally, we resected a lesion, although a tiny residue remained on the margin of the lesion, which was resected by a snare (Figs. 1F and G). The pathologic diagnosis was low-grade tubular adenoma (Fig. 1H). Follow-up colonoscopy 6 months later revealed that the post-ESD ulcer had become completely scarred without residual lesion (Fig. 1I).

ESD is still technically difficult for lesions with severe fibrosis. In this case, use of ESD managed to avoid the need for repetitive surgery by highly skillful hands and multiple devices. When perforation occurs during ESD, we attempt to close the perforation site using endoscopic clipping; if that fails, conversion to surgery is necessary. When endoscopic clipping succeeds, localized peritonitis is managed with conservative care, including fasting and intravenous antibiotic administration, but if generalized peritonitis occurs, again, conversion to surgery may be needed. In any case, consultation with a surgeon is necessary, so it is important to maintain good relationships with surgeons.

\section{DISCLOSURE}

All authors disclosed no financial relationships relevant to this publication.

Michiko Wada, MD, Motohiko Kato, MD, PhD, Yuichiro Hirai, MD, Toshio Uraoka, MD, PhD, Department of Gastroenterology, National Hospital Organization Tokyo Medical Center, Tokyo, Japan

Copyright (c) 2017 American Society for Gastrointestinal Endoscopy Published by Elsevier Inc. This is an open access article under the CC BY-NC-ND license (http://creativecommons.org/licenses/by-nc-nd/4.0/).

http://dx.doi.org/10.1016/j.vgie.2017.02.001 